\title{
A Novel Region Based Liveness Detection Approach for Fingerprint Scanners
}

\author{
Brian DeCann, Bozhao Tan, and Stephanie Schuckers \\ Clarkson University, Potsdam, NY 13699 USA \\ \{decannbm, tanb, sschucke\}@clarkson.edu
}

\begin{abstract}
Biometric scanners have become widely popular in providing security to information technology and entry to otherwise sensitive locations. However, these systems have been proven to be vulnerable to spoofing, or granting entry to an imposter using fake fingers. While matching algorithms are highly successful in identifying the unique fingerprint biometric of an individual, they lack the ability to determine if the source of the image is coming from a living individual, or a fake finger, comprised of PlayDoh, silicon, gelatin or other material. Detection of liveness patterns is one method in which physiological traits are identified in order to ensure that the image received by the scanner is coming from a living source. In this paper, a new algorithm for detection of perspiration is proposed. The method quantifies perspiration via region labeling methods, a simple computer vision technique. This method is capable of extracting observable trends in live and spoof images, generally relating to the differences found in the number and size of identifiable regions per contour along a ridge or valley segment. This approach was tested on a optical fingerprint scanner, Identix DFR2100. The dataset includes a total of 1526 live and 1588 spoof fingerprints, arising from over 150 unique individuals with multiple visits. Performance was evaluated through a neural network classifier, and the results are compared to previous studies using intensity based ridge and valley liveness detection. The results yield excellent classification, achieving overall classification rates greater than $95.5 \%$. Implementation of this liveness detection method can greatly improve the security of fingerprint scanners.
\end{abstract}

Keywords: Biometrics, fingerprint, liveness detection, neural network.

\section{Introduction}

Biometric systems are becoming popularized for their ability to grant security to restricted individuals, without them needing to carry small objects such as keys, or memorize long and abstract passwords [1. However, fingerprint scanners have been shown to be vulnerable to attack at the sensor level, granting access to well made spoof fingers, or even dismembered live fingers [2. Liveness detection is a measure that determines whether or not the source of the image presented to a biometric scanner is from a living individual. Its purpose is to provide extra security to the system by working cooperatively with matching algorithms that recognize an enrolled user.

M. Tistarelli and M.S. Nixon (Eds.): ICB 2009, LNCS 5558, pp. 627-636, 2009.

(C) Springer-Verlag Berlin Heidelberg 2009 


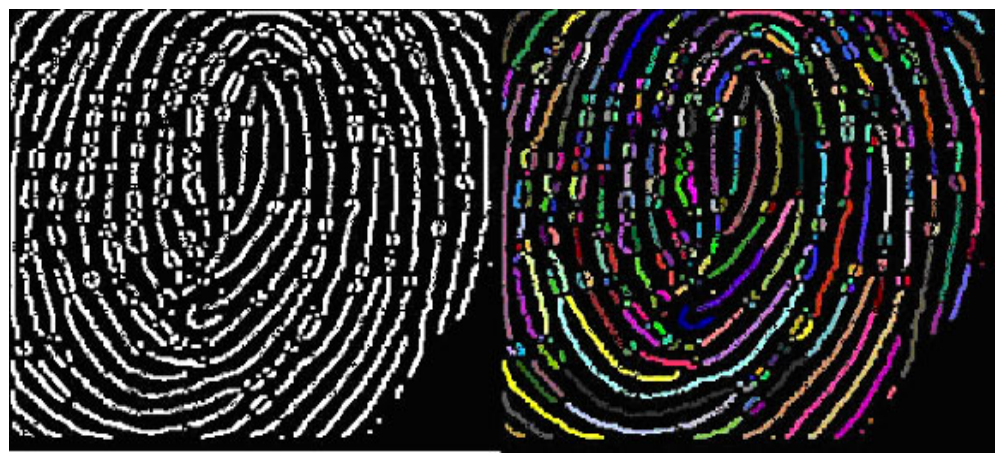

Fig. 1. Region Labeling of a Fingerprint Image. Left) Binary image with foreground (white) and background (black) pixels. Right) Labeled image. Note each region is assigned a different color.

Software-based liveness detection has been an active research area in recent five years, which uses the information already captured by traditional fingerprint systems to detect life signs, for example, skin deformation or elasticity, perspiration pattern or characteristics of spoof and live images. Skin deformation [34 5 6] technique uses the information regarding how the fingertip's skin deforms when pressed against the scanner surface. Perspiration pattern detection method [13147] quantifies the perspiration phenomenon inherent only to live fingers due to the existence of pores and perspiration using two time series images or a single image. Also researchers investigate the characteristics of spoof and live fingers, like power spectrum [12] or band-selected Fourier Spectrum [9], skin coarseness [11, valley noise [15], gray-level profile and frequency patterns [8] or integration of multiple static features [10].

The aim of this study is to build upon the previous research and propose a new liveness detection method, region labeling technique, which provides different characteristics of live and spoof features which may improve upon classification. In general, region labeling is a simple computer vision technique, which identifies areas of foreground from the background. Refer to the following figure for an example. Here, region labeling can be applied to quantify observable differences between live and spoof images. Regarding the capture of a single image, there exists more identifiable regions along both ridge and valley segments than contained in spoof images. This would be expected because ridge segments in live images tend to have consistent intervals of high and low intensity, corresponding to pore locations, a trait not common in spoof images. In the valleys, spoof images are often prone to pressure deformation, causing ridge segments to bleed into the valley. Live images remain relatively clean, though due to the effects of noise, several small areas of intensity should be present. Difference images, a type of image where two captures are subtracted from one another can also expose traits which can be exploited by region labeling. In this format, the element of control an imposter has over a fingerprint scanner is exposed. Here, the slight variance of pressure and rotation distorts ridge segments which would otherwise 
remain intact, resulting in more identifiable regions in spoof images than live images. Additionally, tracking region size can also be taken into account to verify this claim. This method is advantageous in that it looks at the perspiration pattern in a different sense, as well as isolating traits of spoof images. Another advantage is simplicity where the required computations are presently available and well known.

\section{Region Labeling Algorithm}

The most naturally evident form of vitality in a fingerprint is its perspiration. Since perspiration can only occur from sweat glands of living tissue, the ability to quantify it makes for an excellent liveness classification method. Fig. 2 illustrates the differences in time between a living fingerprint, and one that has been spoofed.

a)
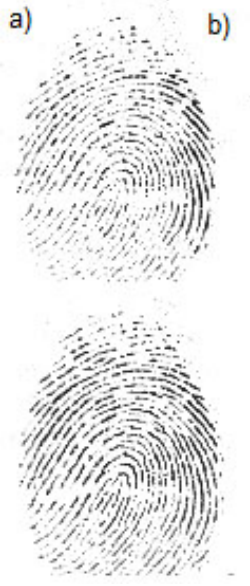

b)
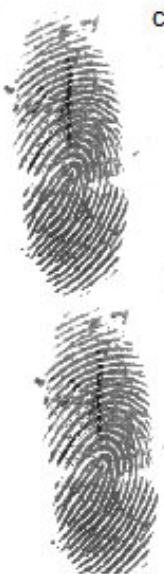

c)
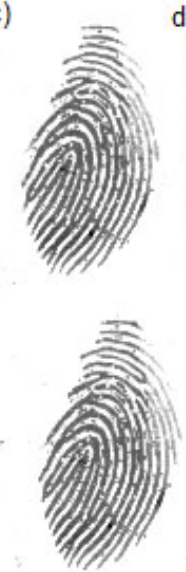

d)
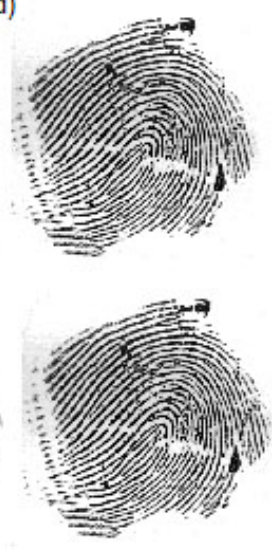

Fig. 2. Fingerprint images for a particular subject. The top row represents time zero, and the bottom 2 seconds following. a) Live, b) Silicone, c) Gelatin, d) PlayDoh.

From Fig. 2, it is seen that the live image darkens, as well as thickens along the ridges with time. The spoof images however, remain relatively consistent, and are noticeably darker in intensity. The valleys of the live image as well, are relatively clean, and free of pixels, while this does not appear to be the case for the spoof images. Furthermore, the lengths of the ridge segments along the live signal are smaller, and spotty in appearance. This differs from spoof images in the sense that the segments for those images are rather long and consistent. From a statistical standpoint, the motives of the previous work are clear, as through observation one can visibly see evident intensity based differences, and expect differences in the frequency domain. 
While these images alone make it difficult to visually witness the perspiration effect, the subtraction of two images, can illustrate this much more efficiently, as expressed in Fig.3.
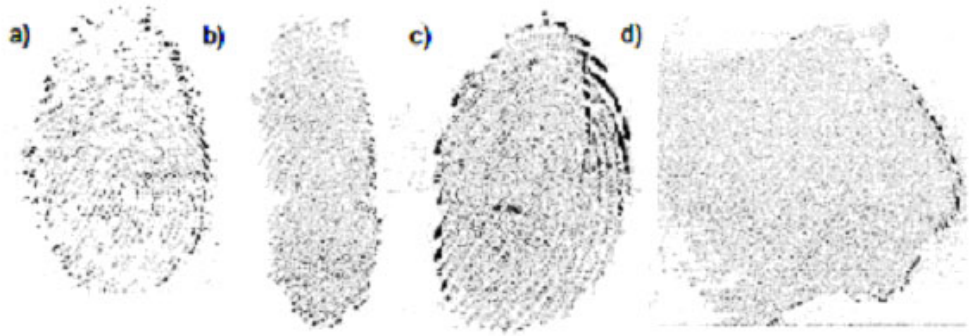

Fig. 3. Absolute difference between images at $t=2$ and $t=0$. a) Live. b) Silicone. c) Gelatin. d) PlayDoh (Darkness indicates significant change).

\subsection{Image Capture and Preprocessing}

Images are captured sequentially at an interval of one second. Difference images are then created from the subtraction of the final image from the initial. Preprocessing of captured images begins with a cropping of a rectangular region whose center is the center of the scanner. This reduces the computational effort to a degree by assuming that the user will not place a piece of their finger along the corners of the scanner itself in an attempt to gain access. The first step of preprocessing the images is to adaptively account for irregularities in regard to the scanner itself. This is done through use of a program that compares the recorded images to a blank capture, and uses a $2 \%$ threshold in which to decide if a pixel should reflect the intensity presented in the image, otherwise it is discarded.

\subsection{Mask Generation and 1-D Signal Extraction}

Veridicom SDK is used to convert the gray image to binary image. Once the image is binarized, a simple thinning algorithm can thin the binarized image down to a width of 1 pixel. The remaining binary image then has all of its $\mathrm{Y}$ intersections removed, so that a signal has a well defined start and endpoint. Following a shift procedure, the resulting contours are aligned with either the center of the ridges or valleys, depending on operation. A length check removes contours that are too small for computation, on the basis that their pixel distance is shorter than the length between pores found on the skin.

\subsection{Region Identification}

At this point, the filtered initial and subsequent images, along with a contour mask, representing pixel locations of the ridges or valleys are prepared for use. In order to account for the entirety of intensity values that may lie within a ridge 
or valley, the contour mask is dilated to a width of three pixels. Fig. 4 illustrates superposition of the mask along both ridge and valley signals, before and after dilation.

The next step is to identify the size and number of regions present along the dilated contours. Here, the methodology diverges into application for both single and double image capture. For multiple image capture, a difference image is created based on pixel intensity change between the initial and secondary capture. Three types of difference images can be observed. Pixel locations whose change is negative reflects an area that has decreased in intensity, or became darker. This would arise from true perspiration, increasing pressure, or movement. Additionally, an image can be created to reflect positive change in pixel values. This sort of intensity change naturally arises from motion. Finally, an image reflecting the absolute difference illustrates the combination of any sort of pixel change.

Each individual contour along the difference image is initially checked for a length threshold. Assuming the thresholding criterion is met, the labeling operation can then be performed on an isolated contour. As a requirement for

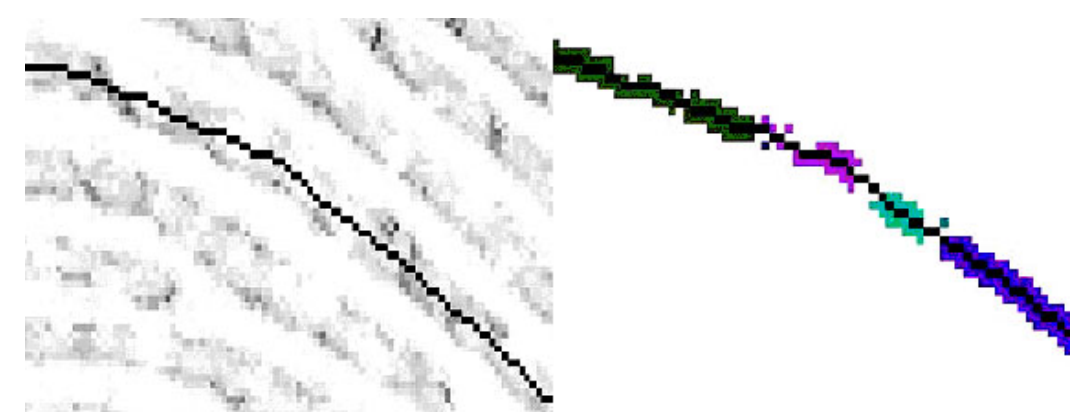

Fig. 4. Live Region Identification. Note the variance of region size. Left) Negative Difference Image with Contour of Interest Colored (Ridge Signal). Right) Labeled Contour of Interest.

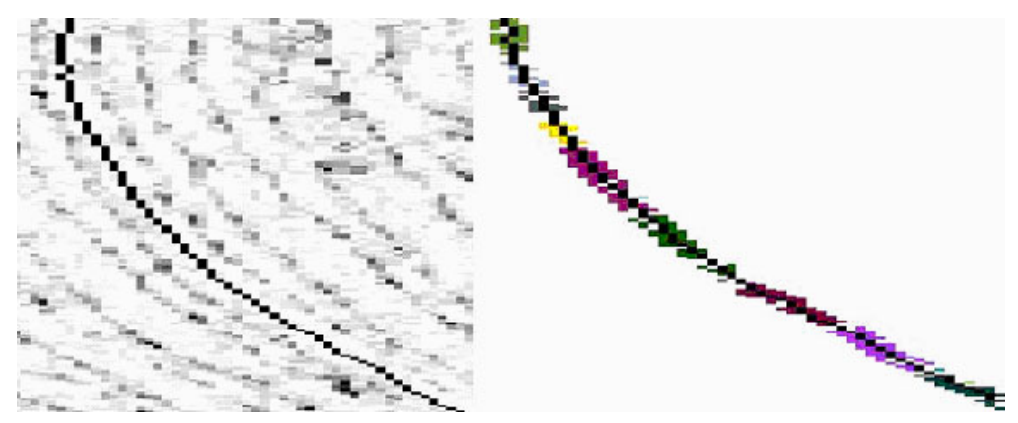

Fig. 5. Spoof Region Identification. Note the consistency of region size. Left) Negative Difference Image with Contour of Interest Colored (Ridge Signal). Right) Labeled Contour of Interest. 
region labeling algorithms, the image itself must first be converted into its respective foreground and background. In this case, the conversion is trivial since the only pixels of interest are those that have changed along the contour being examined. The labeling algorithm itself is very simple. It operates by isolating an individual pixel, and any 8 connected neighborhood pixels are assigned a number corresponding to its region identifier. The result of this labeling operation, along with a newly labeled image, is the total number of regions found within the contour itself. A visual of the labeling process is process is provided in Fig. 4 and 5.

Fig. 4 and 5 help to illustrate a specific property in classification of live and spoof images. The live image for example, shows about 6 regions of differing size. Also evident are the gaps between each region. These gaps are indicative of pore locations. The spoof image in Fig. 5 however, has many more regions, with the majority smaller in size than represented in Fig. 4. The lack of gaps, as evident in Fig. 4, illustrates how pressure deformation removes pore locations, and instead replaces them with regions with a size of a few pixels.

For single image capture, the only difference is the steps involving the process to convert the image into a binary format. In this case, the pixels of interest are those that are deemed to be within a specific tolerance range. In the algorithm, foreground pixels are determined by being within $90 \%$ of the maximum intensity value along the contour of interest. This rationality for the threshold is to prevent pixels that have weak intensity values to contribute, and possibly join adjacent regions.

\subsection{Feature Extraction}

Following the labeling procedure for each specific image type, statistical properties can be extracted. Features include the total number of regions found (per image), regions found per contour, average region size (in pixels) and the standard deviation of region size. Use of the time series difference images (absolute, negative, positive) and the first capture images yield a total of 16 total measures. Recalling that the measures can be repeated for both ridge and valley analysis results in potentially 32 region based measures available for classification.

Fig. 6 illustrates a few examples of features extracted between live and spoof fingerprints. As we can see from Fig. 6-a, spoof difference images on average contain more regions per contour along ridge segments, and fewer along the valleys, when compared to a live difference image, supporting the original claim. As previously mentioned, this occurs as a result of the lack of control an imposter has over a fingerprint scanner when using a spoof. Minor inconsistencies in the ridge segment are reflected as pixel change and will subsequently result in a higher region count. As there is little apparent change in the valley of a live image (Fig. 2-a), the effects of noise result in several small regions, which are typically absorbed in a spoof image due to unintended pressure deformation (Fig 3-b,c,d). Fig. 6-b aims to measure the consistency of region size in ridge segments. As can be inferred, region size in spoof images is much less random than live images. This result continues to support the image presented in Fig. 5, 


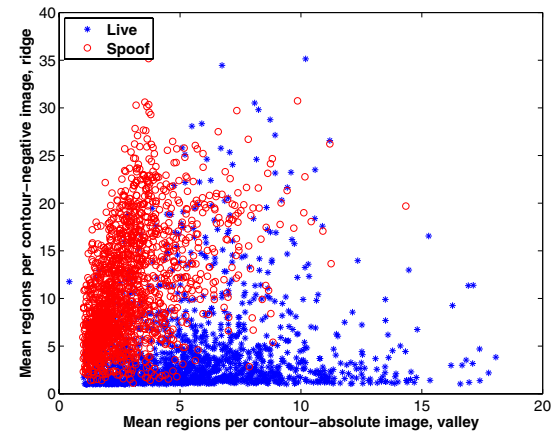

(a) Mean regions per contour, valley absolute image vs ridge negative image

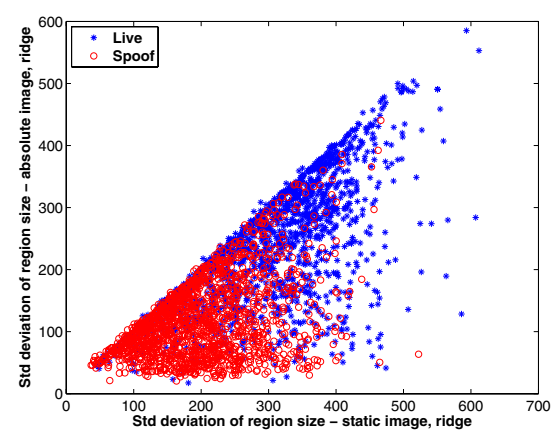

(b) Std deviation of region size, ridge static image vs ridge absolute image

Fig. 6. Feature comparison between live and spoof fingerprints

where the illustrated contour contains many small regions with little variation. In a single image, the result verifies the randomness property of region size in a live image because pixel intensity is much more variant (Fig. 2-a).

\subsection{Classification}

After region labeling features are extracted, standard pattern classification tools (neural network, nearest neighbour, classification tree, etc.) can be used to separate non-live subjects from the live subjects. The non-live category includes the spoof data made from Play-Doh, gelatin and silicone fingers. The software we used for classification is from WEKA (Waikato Environment for Knowledge Analysis) software tool [16] that provides different classification techniques for large data sets. 10 -fold cross-validation test was used to test the new method.

\section{Data Collection}

Identix DFR 2100 was used to collect all fingerprint data for this study. Time series data of at $0 \mathrm{~s}$ and $1 \mathrm{~s}$ are primarily used. The International Review Board (IRB) at A University approved all protocols for data collection. Fingerprints that are collected consist of the Right thumb and Right index fingers. There are 160 live subjects involved in the data collection, each subject with average of 4.6 visits with an average of one month between visits.

In order to create the Spoof fingers, the material which is used to create a spoof finger is placed and conformed to a fingerprint cast created from dental impression materials, which contains the fingerprint of an individual. Spoof images are created from common household items such as PlayDoh, Gelatin (Knox brand), and commercial grade Silicone (Dow Corning Sylgard 184 Silicone Elastomer Kit). Spoof variability is introduced into the spoof library through time. 
In the process of collecting data, several images are created of the same spoof material over approximately ten minutes as the spoof materials dry.

In total, we have collected 1526 live images, 800 Play-Doh, 649 gelatin and 139 silicone spoof fingerprints.

\section{Experiments and Results}

\subsection{Performance Evaluation}

To evaluate our system, we utilize terms for evaluating biometric identification or verification systems. Liveness classification is the process of determining whether the input finger is live or spoof.

Because of the threshold selection or decision logic, liveness classification makes two type of errors, i.e., spoof false acceptance (SFA), where a spoof finger is accepted as live and live false rejection (LFR), where a live finger is rejected. Two other terms are also often used, true acceptance, where a live finger is recognized as live and true rejection, where a spoof finger is recognized as spoof. Normalized SFA and LFR are used, which are called spoof false acceptance ratio (SFAR) and live false rejection ratio (LFRR), respectively. They can be calculated as

$$
\begin{aligned}
& S F A R=\frac{S F A}{N S} \\
& L F R R=\frac{L F R}{N L}
\end{aligned}
$$

where SFA and LFR is the number of SFA and LFR samples, respectively; and NS and NL are the total number of spoof finger samples and live finger samples, respectively. Equal error rate (EER) is when SFAR equals LFRR. To evaluate the performance of our system, we use Receiver Operator Characteristic (ROC) curve, which plots the change of true positive rate (1-LFRR) with SFAR for varying thresholds.

\subsection{Results and Discussion}

10-fold cross-validation was used to evaluate the performance of the proposed algorithm. To make a comparison, we also implemented the liveness detection algorithms described in 1415. Ridge signal algorithm 13. quantifies the perspiration pattern along the signal along ridges, while valley noise algorithm [15] characterize the difference between live and spoof fingerprints along valleys. Fig. 7 shows the ROC curve. As seen from this figure, the new region label technique can reach an EER of $4.5 \%$, while ridge signal algorithm can reach $5.1 \%$ and valley noise algorithm can reach $3.2 \%$, respectively. Also, the fusion of three approaches is also implemented at feature level, the outcome can reach an EER of as low as $1.2 \%$. The proposed method explores the perspiration pattern in more detail, investigating the positive, negative and absolute difference images 


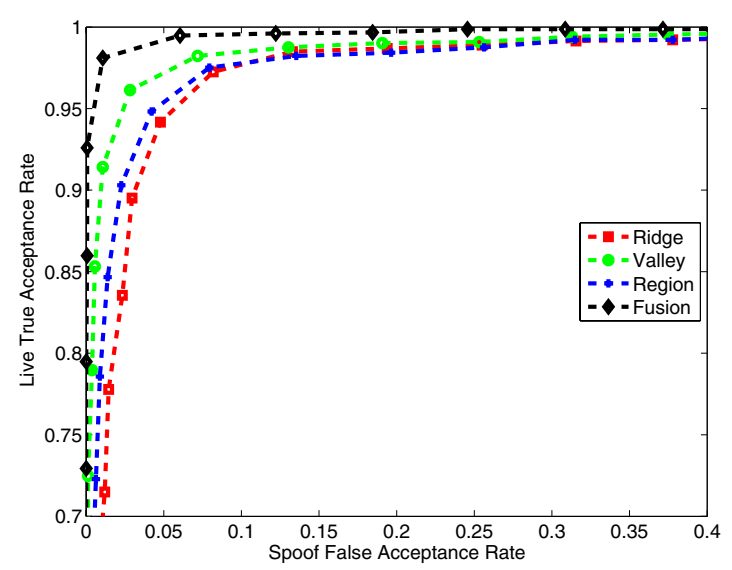

Fig. 7. ROC comparison between ridge signal algorithm, valley noise algorithm and new region label algorithm

between 0 and 1 second images, in addition to the first single image. The addition of the new region label technique can be used to fuse with ridge signal or valley noise algorithms to improve the overall liveness classification.

Furthermore, another standout feature of these measures is that they do not require great lengths of computation. The majority of the calculations are simple logical relations (thresholding), basic mathematical operations (creating difference images), and use of basic image processing algorithms.

Future work includes testing on other types of scanners, collecting more live data with environmental changes, like temperature or humidity. Also it would be necessary to investigate more spoofing techniques, like trying different spoof materials or techniques, which can cause a spoof finger to break the algorithm. These information will be used to tune the proposed algorithm to a more robust liveness classification agent.

\section{Conclusion}

This study demonstrated a new method to quantify liveness detection through region labeling of the first capture and three difference images of first and second capture (absolute, positive and negative). This approach differs from previous methods in the sense that it aims to interpret liveness based on its visual characteristics that characterize live and spoof images. The test database for this study consisted of over 1,500 live and spoof images for Identix fingerprint scanner. The size of the database helps to ensure there exists sufficient testing variability, to encompass situations that would arise in everyday use. 


\section{References}

1. Jain, A., Bolle, R., Pankanti, S.: Biometrics: Personal Identification in Networked Society. Springer, Heidelberg (1999)

2. Schuckers, S.A.C.: Spoofing and anti-spoofing measures. Information Security Technical Report 7(4), 56-62 (2002)

3. Maltoni, D., Maio, D., Jain, A., Prabhakar, S.: Handbook of Fingerprint Recognition. Springer, $\mathrm{Nu}(2003)$

4. Antonelli, A., Cappelli, R., Maio, D., Maltoni, D.: Fake Finger Detection by Skin Distortion Analysis. IEEE Transactions on Information Forensics and Security 1(3), 360-373 (2006)

5. Chen, Y., Jain, A., Dass, S.: Fingerprint Deformation for Spoof Detection. In: Proc. of Biometrics Symposium (BSYM 2005), Arlington, VA, September 19-21 (2005)

6. Zhang, Y., Tian, J., Chen, X., Yang, X., Shi, P.: Fake Finger Detection Baed on Thin-late Spline Distortion Model. In: Lee, S.-W., Li, S.Z. (eds.) ICB 2007. LNCS, vol. 4642, pp. 742-749. Springer, Heidelberg (2007)

7. Jia, J., Cai, L.: Fake Finger Detection Based on Time-series Fingerprint Image Analysis. In: Huang, D.-S., Heutte, L., Loog, M. (eds.) ICIC 2007. LNCS, vol. 4681, pp. 1140-1150. Springer, Heidelberg (2007)

8. Uchida, K.: Image-Based Appraoch to Fingerprint Acceptability Assessment. In: Zhang, D., Jain, A.K. (eds.) ICBA 2004. LNCS, vol. 3072, pp. 294-300. Springer, Heidelberg (2004)

9. Jin, C., Kim, H., Elliott, S.: Liveness Detection of Fingerprint Based on BandSelective Fourier Spectrum. In: Nam, K.-H., Rhee, G. (eds.) ICISC 2007. LNCS, vol. 4817, pp. 168-179. Springer, Heidelberg (2007)

10. Choi, H., Kang, R., CHoi, K., Kim, J.: Aliveness Detection of Fingerprints using Multiple Static Features. In: Proc. of World Academy of Science, Engineering and Technology, July 2007, vol. 22 (2007)

11. Moon, Y.S., Chen, J.S., Chan, K.C., So, K., Woo, K.C.: Wavelet based fingerprint liveness detection. Electronic Letters 41(20), 1112-1113 (2005)

12. Coli, P., Marcialis, G.L., Roli, F.: Power spectrum-based fingerprint vitality detection. In: IEEE Workshop on AutoID (June 2007)

13. Derakhshani, R., Schuckers, S.A.C., Hornak, L., O'Gorman, L.: Determination of vitality from a noninvasive biomedical measurement for use in fingerprint scanners. Pattern Recognition 36(2) (2003)

14. Tan, B., Schuckers, S.: Liveness Detection for Fingerprint Scanners Based on the Statistics of Wavelet Signal Processing. In: IEEE CVPRW (June 2006)

15. Tan, B., Schuckers, S.: A New Approach for Liveness Detection in Fingerprint Scanners Based on Valley Noise Analysis. SPIE Journal of Electronic Imaging 17(1) (2008)

16. Witten, I.H., Frank, E.: Data Mining: Practical machine learning tools and techniques, 2nd edn. Morgan Kaufmann, San Francisco (2005) 